\title{
Research at the Biomedical Analysis Group of the University Utrecht, The Netherlands
}

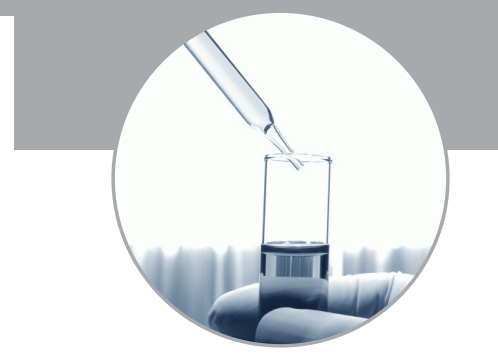

\author{
"Driven by analytical questions from biomedical research and development, the Biomedical Analysis Group will continue to \\ design novel, improved and complementary techniques for pharmaceutical analysis and bioanalysis."
}

The Biomedical Analysis Group is part of the Department of Pharmaceutical Sciences at Utrecht University (UU), The Netherlands. The group participates in the Academic Biomedical Centre, which unifies the strong expertise in life science and biomolecular research at $\mathrm{UU}$ in the fields of (veterinary) medicine, pharmacy, biology and chemistry. The UU has denoted drug innovation as one of its major focus areas.

The Biomedical Analysis Group designs and applies novel analytical methodologies for pharmaceutical and biomedical purposes. Requirements in the pharmaceutical, biomedical and clinical fields are starting points for our research strategy, which is directed towards the investigation of innovative analytical concepts with particular attention on sample pretreatment, separation and detection using state-ofthe-art instrumentation. The group's research focuses on advanced separation techniques and their combination with MS. Special emphasis is put on capillary electromigration techniques and nano-LC. The group explores the wide potential of CE for achieving new or improved possibilities for drug and biomolecular analysis. CE is a microscale technique providing fast and efficient separations and requiring only very small amounts of sample and solvents. The mechanism of CE offers unique selectivity and separation performance. CE is also particularly useful for highly polar compounds that may not be easily covered by other separation techniques. The potential of monolithic materials for nano-LC to accomplish fast and efficient separations for peptides and proteins is another research theme.

The group's research started in 1995 at the University Centre for Pharmacy in Groningen, The Netherlands, with the development of new methods for drug-impurity profiling based on CE. The efforts quickly expanded to the analysis of new protein therapeutics and DNA (genotyping). The move of the authors to UU in 2001 strongly boosted the development and use of new CE-MS methodologies, in which the group now holds a leading international position. Method development has been directed towards (chiral) drugs, peptides, (glyco) proteins, carbohydrates and metabolites and has triggered various collaborations with the pharmaceutical industry, hospitals, instrument companies and academic groups. At present, our particular research focus is on drug profiling, characterization of protein pharmaceuticals and metabolomics. The main lines of research are outlined in the next section.

\section{Research profile}

\section{- Drug profiling by CE-MS}

CE is very suitable for the analysis of ionogenic drugs and their impurities. The separation mechanism of its most widely used mode, capillary zone electrophoresis, allows analyte differentiation according to charge-to-size ratio, thus yielding valuable orthogonality with common separation techniques such as reversed-phase LC. Special attention is paid to electrokinetic chromatography (EKC) as both charged and neutral drug compounds can be separated. EKC methods often involve the use of nonvolatile pseudo-stationary phases, such as micelles and cyclodextrines, which may interfere with ESI. Several successful EKC-ESI-MS approaches have been developed and their applicability for impurity profiling of drugs was demonstrated (Figure I). The group was one of the first to introduce alternative ionization techniques, such as atmospheric pressure photoionization, to CE-MS and EKC-MS. Unlike ESI, these techniques do not suffer from signal suppression by nonvolatile constituents and are particularly useful for less polar compounds. The different ionization techniques have been evaluated systematically and were shown to provide complementary information on drug impurities.

\section{GJ de Jong ${ }^{\dagger 1}$ \& GW Somsen ${ }^{1,2}$}

'Biomedical Analysis, Department of Pharmaceutical Sciences, Faculty of Science, University Utrecht,

The Netherlands

${ }^{2}$ Tel.: +31 302536951

E-mail: gw.somsen@uu.nl

${ }^{\dagger}$ Author for correspondence:

Tel.: +31 302536591

E-mail: g.j.dejong@uu.nl 


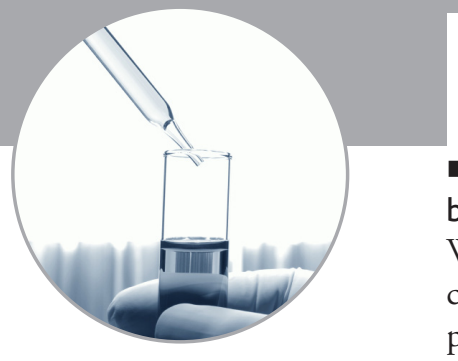

- Characterization of biopharmaceuticals by CE-MS \& CE-fluorescence

When compared with conventional drugs, the characterization of protein pharmaceuticals poses a great challenge due to their complex properties. Proteins may comprise different isoforms and highly related impurities. Protein structural integrity is essential for activity and, therefore, degradation, protein (un)folding and formation of aggregates should preferably be monitored. CE is an attractive tool for the

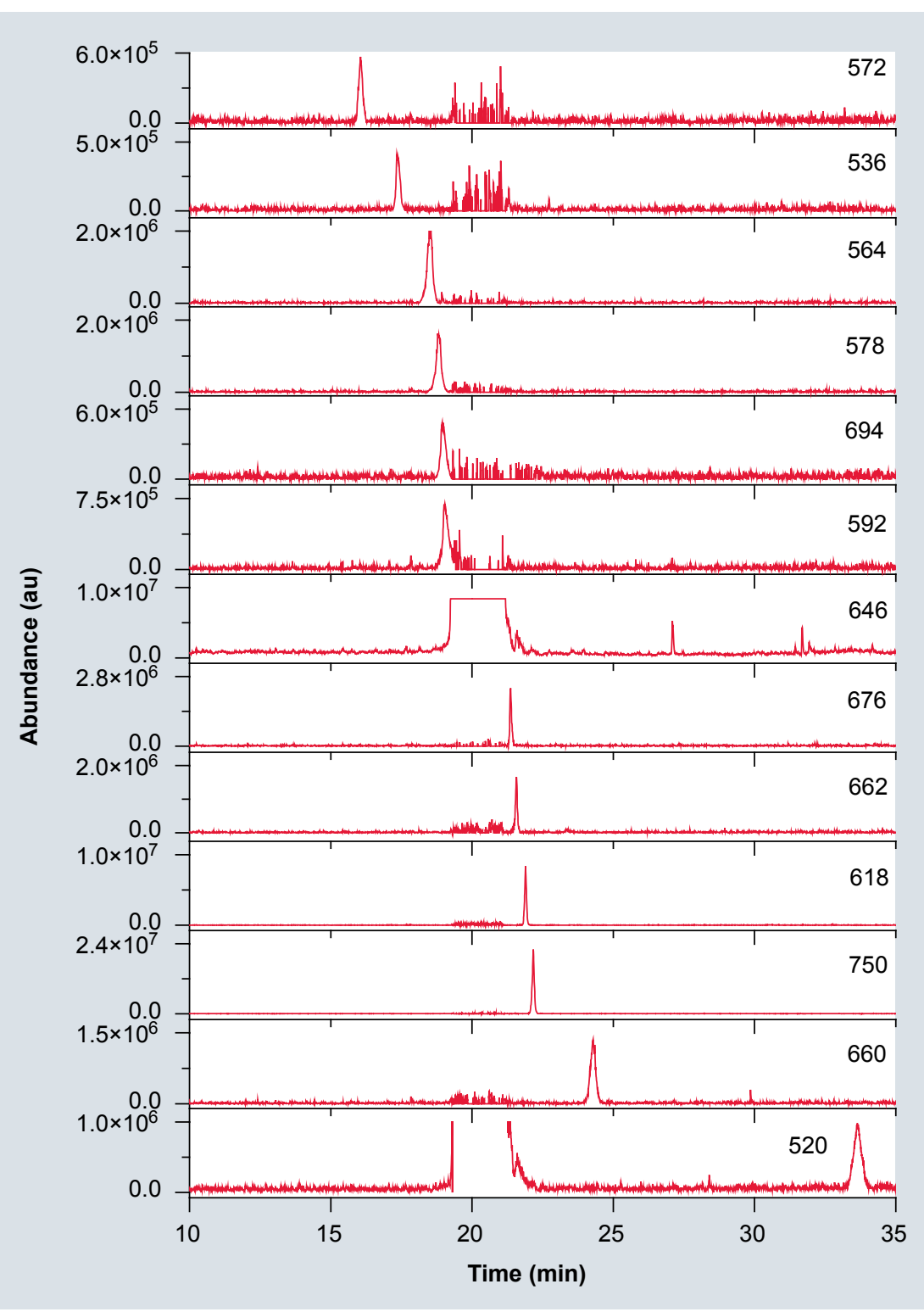

Figure 1. Impurity profiling of the drug amiodarone by nonaqueous electrokinetic chromatography with ESI-MS detection. Eextracted-ion chromatograms at indicated $\mathrm{m} / \mathrm{z}$ values are shown. A total of 12 impurities are revealed with an overall relative content of $0.47 \%$ (area/area). Background electrolyte: $10-\mathrm{mM}$ ammonium formate, $0.75-\mathrm{M}$ formic acid and 10-mM HDAS- $\beta$-cyclodextrin in methanol.

Reproduced with permission from [1] @ Wiley-VCH Verlag GmbH \& Co. KGaA. separation of intact proteins, offering fast and efficient separations. Moreover, CE can be conducted in 'biocompatible' buffers under conditions that allow analysis of proteins in their native state.

Methodologies for efficient CE-MS of proteins have been developed by the group. Capillary-coating strategies involving noncovalently bound polymers are used to prevent the adverse adsorption of proteins to the capillary wall. TOF-MS, which presents the high mass resolution and accuracy needed for native protein analysis, has been coupled to CE, providing the possibility of identifying highly related degradation products in biopharmaceuticals (FIGUre 2). CE-MS interfacing schemes, with and without the use of sheath liquids, are designed and compared for protein analysis in order to achieve sub-nanomolar sensitivities. The coupling of capillary isoelectric focusing (CIEF) and MALDI-MS using spotting technologies has been accomplished and applied to the analysis of biopharmaceuticals. Another line of research involves the development of selective wavelength-resolved fluorescence detection of protein pharmaceuticals in CE. Making use of native protein fluorescence, information on protein purity and conformation can be obtained simultaneously.

\section{SPE-CE}

The concentration sensitivity of $\mathrm{CE}$ is relatively low, due to the intrinsically small injection volumes (1-100 nl). The usefulness of CE for the analysis of low-abundant compounds in biofluids can be greatly improved by applying electrophoretic and/or chromatographic preconcentration techniques. The group has paid particular attention to the online and inline combination of SPE with CE. In the online mode, the SPE column has been coupled to the $\mathrm{CE}$ system using new valve-based interfacing techniques providing solvent-switching capabilities. In the inline mode, the SPE material is an integrated part of the CE capillary, avoiding dead volumes and loss of analyte; however, the introduction of the biological matrix into the capillary can disturb the separation. Both coupling approaches have been evaluated and applied to the determination of neuropeptides in CSF, yielding detection limits in the low nanogram/milliliter range. Powerful bioanalysis is achieved by combining SPE-CE with MS comprising sample handling, separation and selective detection in one hyphenated system (FIGURE 3). 


\section{- Metabolomics by CE-MS}

The metabolome is the complete set of endogenous low-molecular weight metabolites in cells, tissues and body fluids and metabolomics is the comprehensive analysis of these metabolites. Metabolomics provides insight into the (patho) physiological state of an individual as endogenous metabolites represent real end points of biochemical pathways. Given that the overall status of an individual is reflected by his or her metabolic state, metabolomics has the potential to have a great impact upon medical practice by providing useful biochemical data.

Since many metabolites display high polarity and water solubility, CE-MS can be an attractive technique for nontargeted metabolic profiling. Our research has been directed towards the design and use of stable CE-MS systems for comprehensive profiling of polar metabolites in urine, CSF and plasma. Strongly improved system reproducibility was achieved by the use of capillaries that are noncovalently coated with charged polymers. Both positively and negatively charged capillaries are employed in order to obtain complete profiles of cationic and anionic compounds. TOF-MS is used to provide the accurate and exact masses required for the distinction and identification of metabolites. The utility of the CE-MS systems has been tested and demonstrated in close collaboration with the Leiden University Medical Center by the analysis of samples of patients suffering from diseases such as urinary tract infection, complex regional pain syndrome and bacterial meningitis. Multivariate data analyses of the CE-MS data are used to reveal diseaserelated metabolites (biomarkers). A typical CE-MS workflow for metabolomics is depicted in Figure 4.

\section{- Monolithic LC columns}

Monolithic LC columns contain a single piece of stationary phase that displays high permeability and low resistance to mass transfer. Monolithic columns are very suitable for the separation of peptides and proteins. Both silicabased and polymeric materials are studied for the separation of protein digests and protein mixtures. Integrated systems based on monolithic columns, immobilized enzymes and MS detection have been developed. Particularly, the possibility of relatively long monolithic columns for the separation of complex protein digests has been demonstrated (FIGURE 5). Short monolithic columns appear to be well suited for the fast analysis of more simple mixtures.

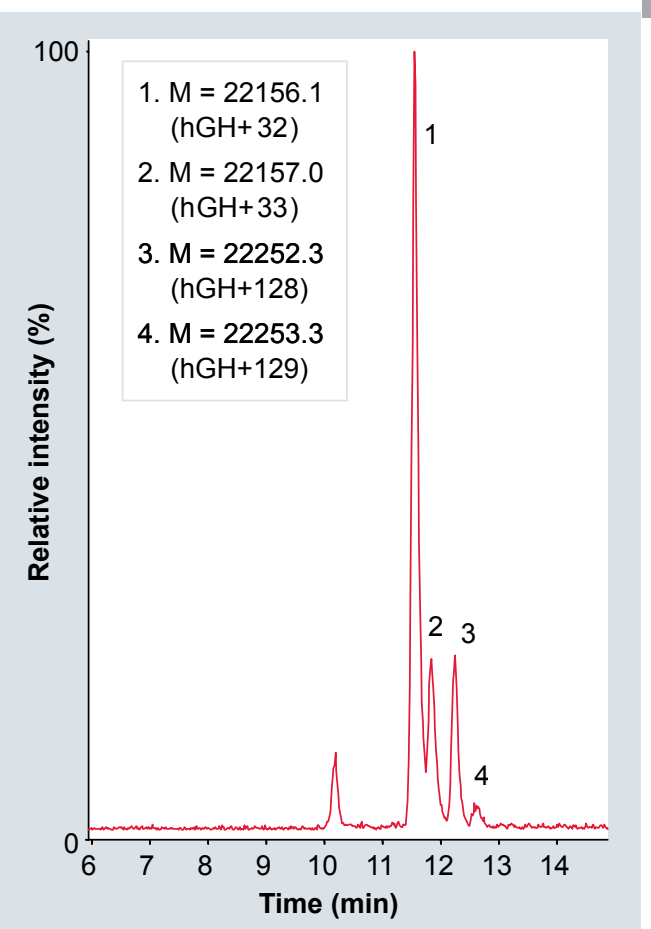

Figure 2. CE-TOF-MS of heat-exposed recombinant human growth hormone. Identified degradation products: $1, \mathrm{hGH}_{-} \mathrm{O}_{2} ; 2$, mono-deamidated hGH-O $; 3$, hGH-O ;

4, mono-deamidated $\mathrm{hGH}_{-} \mathrm{O}_{8}$. Conditions: CE coating, PB-PVS bilayer, BGE 75-mM ammonium formate ( $\mathrm{pH}$ 8.5).

hGH: Human growth hormone.
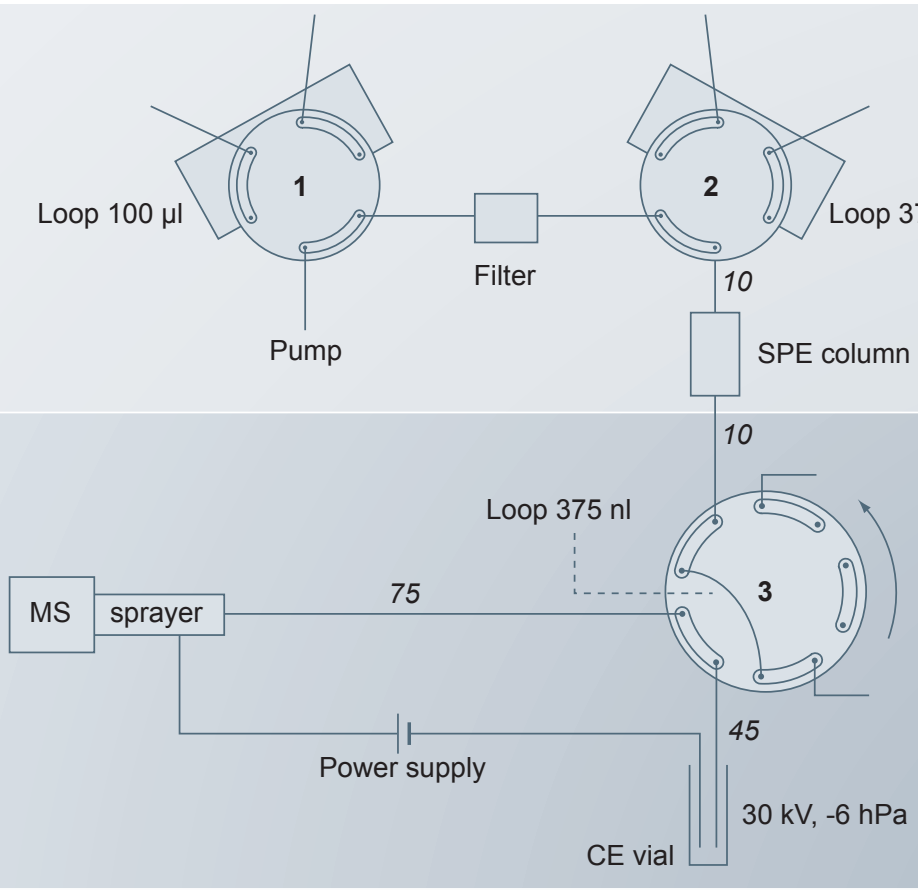

Figure 3. Scheme of online SPE-CE-MS. Lengths (in $\mathrm{cm}$ ) of fused-silica capillaries are shown in italics. All valves are depicted in the load position. Valve 3 is switched from load to inject as indicated by the arrow.

Reproduced with permission from [2] $\odot$ Wiley-VCH Verlag GmbH \& Co. KGaA. 


\section{News \& Analysis | Research SpotLight}
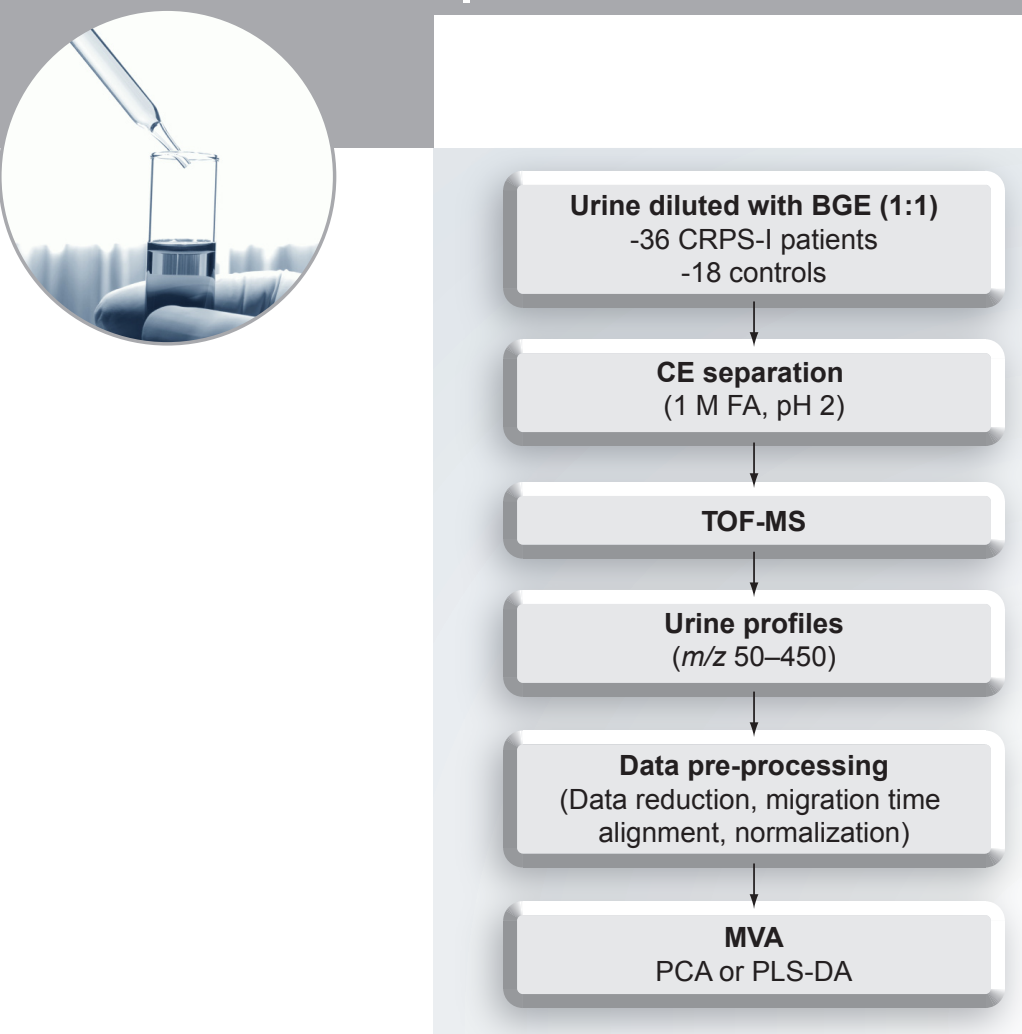

Figure 4. CE-MS workflow for metabolomics study of urine of patients with CRPS. CE capillary coated with polybrenepoly(vinylsulfonate).

BGE: Background electrolyte; CRPS: Complex regional pain syndrome; DA: Discriminant analysis; FA: Formic acid; MVA: Multivariate analysis; PCA: Principle component analysis; PLS: Partial least squares.

Peak capacities of different columns are compared with MS identification scores such as the number of identified peptides and protein sequence coverages.

\section{Future perspective}

Driven by analytical questions arising from biomedical research and development and together with partners from the pharmaceutical industry and institutes, the Biomedical Analysis Group will continue to design novel, improved and complementary techniques for pharmaceutical analysis and bioanalysis. New challenges in drug profiling, such as the determination and identification of genotoxic and immunogenic impurities, will require highly specific and sensitive analytical methodologies. Protein therapeutics will play an increasingly important role in contemporary drug development and production. As a result, there is a growing demand for separation-detection methodologies that allow quality assessment of intact proteins. New analytical approaches are needed that allow the accurate and reliable determination of the potential differences among biosimilars (i.e., generic therapeutic proteins) and the characterization of new biopharmaceuticals, such as therapeutic antibodies, vaccines and drug-carrier constructs. We will develop CE methodologies for these purposes applying

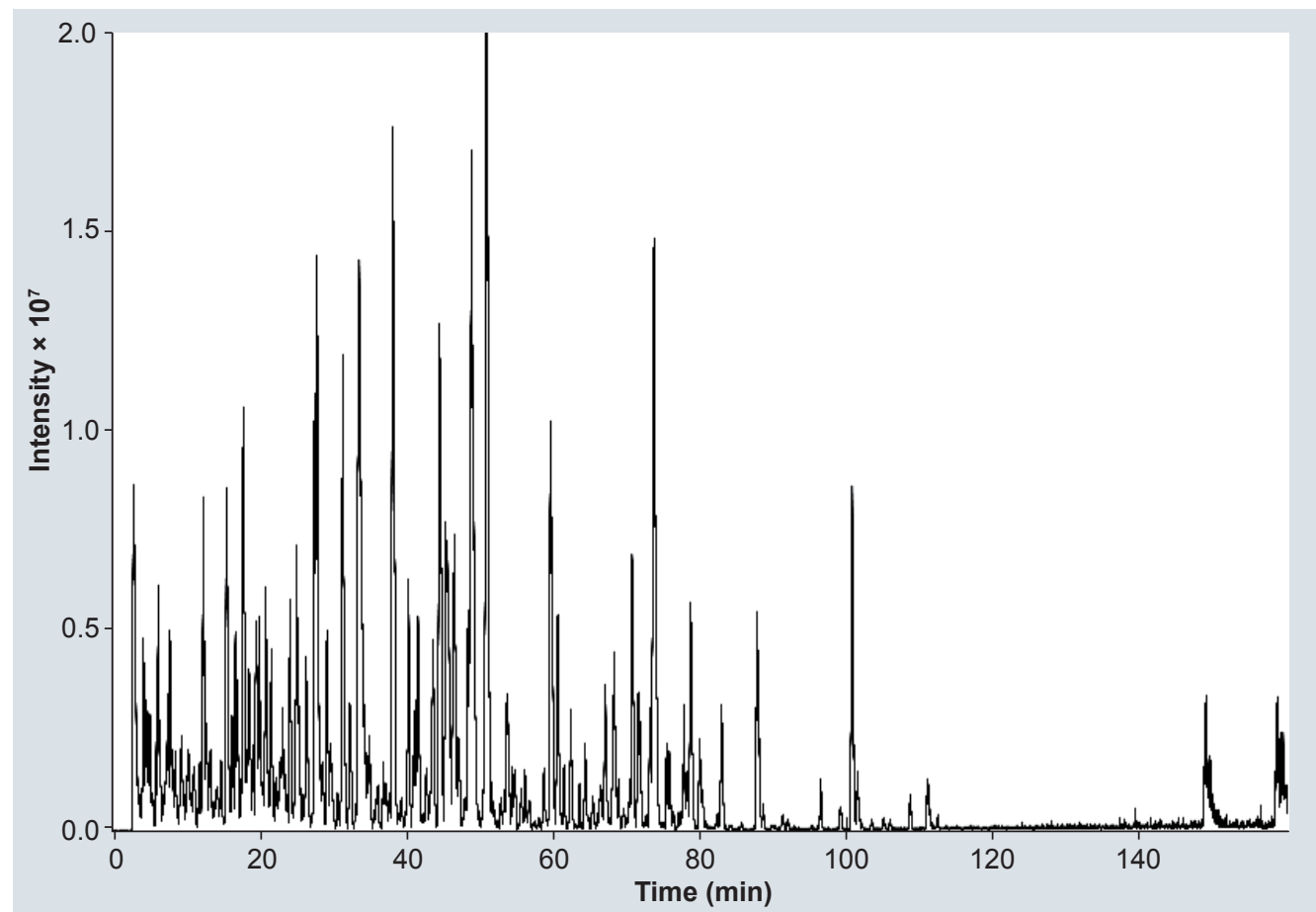

Figure 5. Base peak chromatograms of a nine-protein digest mixture. Separated on polystyrene-divinylbenzene monolithic $250 \times 0.2-\mathrm{mm}$ internal diameter column using a 150-min gradient of $1-31 \%$ acetonitrile in water (both acetonitrile and water containing $0.5 \%$ formic acid). 
appropriate interfacing schemes to combine different $\mathrm{CE}$ modes with advanced mass analyzers and fluorescence detectors. Next to ESI, other ionization techniques, such as MALDI, will be used and optimized for CE-MS, providing high sensitivity and structural information for target compounds.

The potential of CE techniques for selective and sensitive bioanalysis of compounds that are difficult to analyze with other methods will be further explored. Particular attention will be paid to the profiling of highly polar metabolites in biofluids, the possibilities of achieving enantioselectivity among endogeneous compounds using chiral selectors and the determination of protein therapeutics in plasma or serum. As the sensitivity of CE-MS is still limited when compared with LC-MS, improvement of detection limits by electrokinetic and chromatographic preconcentration will be further investigated. Selective SPE using (bioaffinity) phases will be pursued and combined with CE and LC. Comprehensive profiling of metabolites will be a major goal using a few CE methods applying appropriate coatings. Comparisons will be made with novel LC stationary phases suited for polar compounds and complementarity will be tested for clinical samples.

\section{Financial \& competing interests disclosure}

The authors have no relevant affiliations or financial involvement with any organization or entity with a financial interest in or financial conflict with the subject matter or materials discussed in the manuscript. This includes employment, consultancies, honoraria, stock ownership or options, expert testimony, grants or patents received or pending, or royalties.

No writing assistance was utilized in the production of this manuscript.

\section{Executive summary}

- The research carried out by the Biomedical Analysis Group of the University Utrecht involves the development and evaluation of advanced separation-detection techniques for pharmaceutical analysis and bioanalysis.

- The group's main area of research is exploring the wide potential of CE-MS, including various ionization modes to achieve new and improved methods for the analysis of (bio)pharmaceuticals.

- Improved resolution and reproducibility in CE is pursued by the use of selective buffer additives, capillary coatings and nonaqueous electrolytes.

- For more sensitive bioanalysis, online approaches for preconcentration using electrokinetic and (affinity) chromatographic principles are developed.

- Nano-LC with monolithic stationary phases is studied and used for protein and peptide analysis.

- Application areas in pharmaceutical analysis are drug profiling and characterization of biopharmaceuticals.

- Bioanalytical application areas are metabolic profiling of biofluids and bioanalysis of (bio)pharmaceuticals.

- The group collaborates intensively with the pharmaceutical industry, instrument companies, hospitals and other academic groups.

- Future challenges include the further improvement of sensitivity and selectivity of CE-MS and (nano-)LC-MS systems and the integration of sample handling, efficient separation and selective detection.

\section{Bibliography}

1 Mol R, de Jong GJ, Somsen GW. Cyclodextrinbased nonaqueous electrokinetic chromatography with UV and mass spectrometric detection: application to the impurity profiling of amiodarone. Electrophoresis 29, 3575-3581 (2008).

2 Tempels FWA, Underberg WJM, Somsen GW, de Jong GJ. On-line coupling of SPE and CE-MS for peptide analysis. Electrophoresis 28, 1319-1326 (2007).

\section{Recent publications}

\section{- Drug profiling by CE-MS}

- Mol R, de Jong GJ, Somsen GW. Atmospheric pressure photoionization for enhanced compatibility in on-line micellar electrokinetic chromatography - mass spectrometry. Anal. Chem. 77, 5277-5282 (2005).

- Mol R, Kragt E, Jimidar I, de Jong GJ, Somsen GW. Micellar electrokinetic chromatographyelectrospray ionization mass spectrometry for the identification of drug impurities. J. Chromatogr. A 843, 283-288 (2006).

- Mol R, de Jong GJ, Somsen GW. Coupling of electrokinetic chromatography to mass spectrometry. In: Electrokinetic Chromatography: Theory, Instrumentation and Applications. Pyell U (Ed.). John Wiley \& Sons, Inc. (2006).

- Hommerson P, Khan AM, de Jong GJ, Somsen GW. Comparison of atmospheric pressure photoionization and ESI for CZE-MS of drugs. Electrophoresis 28, 1444-1453 (2007). 
Hommerson P, Khan AM, Bristow T, Niessen W, de Jong GJ, Somsen GW. Photon-independent gas-phase-ion formation in capillary electrophoresis-mass spectrometry using atmospheric pressure photoionization. Anal. Chem. 79, 5351-5357 (2007).

- Mol R, de Jong GJ, Somsen GW. Coupling of non-aqueous electrokinetic chromatography using cationic cyclodextrins with electrospray ionization mass spectrometry. Rapid Commun. Mass Spectrom. 22, 790-796 (2008).

- Mol R, de Jong GJ, Somsen GW. Cyclodextrinbased nonaqueous electrokinetic chromatography with UV and mass spectrometric detection: application to the impurity profiling of amiodarone. Electrophoresis 29, 3575-3581 (2008).

- Hommerson P, Khan AM, de Jong GJ, Somsen GW. Capillary electrophoresis-atmospheric pressure chemical ionization-mass spectrometry using an orthogonal interface: set-up and system parameters. J. Am. Soc. Mass Spectrom. 20, 1311-1318 (2009).

- Hommerson P, Khan AM, Bristow T, Harrison MW, de Jong GJ, Somsen GW. Drug impurity profiling by capillary electrophoresis/mass spectrometry using various ionization techniques. Rapid Comm. Mass Spectrom. 23, 2878-2884 (2009).

\section{- Characterization of biopharmaceuticals by CE-MS \& CE-fluorescence}

- Catai JR, Tervahauta HA, de Jong GJ, Somsen GW. Noncovalently bilayer-coated capillaries for efficient and reproducible analysis of proteins by capillary electrophoresis. J. Chromatogr. A 1083 , 185-192 (2005).

- Catai JR, Sastre Torano J, Jongen P, de Jong GJ, Somsen GW. Analysis of recombinant human growth hormone by capillary electrophoresis with bilayer-coated capillaries using UV and MS detection. J. Chromatogr. B 852, 160-166 (2007) .

- Haselberg R, de Jong GJ, Somsen GW. Capillary electrophoresis-mass spectrometry for the analysis of intact proteins. J. Chromatogr. A 1159, 81-109 (2007).

- Silvertand LHH, Sastre Torano J, de Jong GJ, van Bennekom WP. Improved repeatability and matrix-assisted desorption/ionization - time of flight mass spectrometry compatibility in capillary isoelectric focusing. Electrophoresis 29, 1985-1996 (2008).

- Haselberg R, de Jong GJ, Somsen GW. Capillary electrophoresis of intact basic proteins using noncovalently triple-layer coated capillaries. J. Sep. Sci. 32, 2408-2415 (2009).

- Haselberg R, van der Sneppen L, Ariese F et al. Effectiveness of charged noncovalent polymer coatings against protein adsorption to silica surfaces studied by evanescent-wave cavity ring-down spectroscopy and capillary electrophoresis. Anal. Chem. 81, 10172-10178 (2009).
- Radenovic DC, de Kort BJ, Somsen GW. Lamp-based native fluorescence detection of proteins in capillary electrophoresis. J. Chromatogr. A, 1216, 4629-4632 (2009).

- Silvertand LHH, Sastre Torano J, de Jong GJ, van Bennekom WP. Development and characterization of cIEF-MALDI-TOF MS for protein analysis. Electrophoresis 30, 1828-1835 (2009).

- Somsen GW, Tak YH, Sastre Torano J, Jongen P, de Jong GJ. Determination of oversulfated chondroitin sulfate and dermatan sulfate impurities in heparin by capillary electrophoresis. J. Chromatogr. A, 1216, (2009) 4107-4112.

- Stigter ECA, de Jong GJ, van Bennekom WP. Development of an on-line SPR-digestionnanoLC-MS/MS system for the quantification and identification of interferon- $\gamma$ in plasma. Biosens. Bioelectron. 24, 2184-2190 (2009).

\section{SPE-CE}

- Theodoridis G, de Jong GJ. Solid-phase microextraction: a new tool in contemporary bioanalysis. Adv. Chromatogr. 231-271 (2005).

- Puig P, Tempels FWA, Borrull F et al. On-line coupling of solid-phase extraction and capillary electrophoresis for the determination of cefoperazone and ceftiofur in plasma. $J$. Chromatogr. B 856, 365-370 (2007).

- Tempels FWA, Underberg WJM, Somsen GW, de Jong GJ. On-line coupling of SPE and CE-MS for peptide analysis. Electrophoresis 28, 1319-1326 (2007).

- Puig P, Tempels FWA, Somsen GW et al. Use of large volume sample stacking in on-line solid-phase extraction-capillary electrophoresis for improved sensitivity. Electrophoresis 29, 1339-1346 (2008).

- Ramautar R, Ratnayake CK, Somsen GW, de Jong GJ. Capillary electrophoresis-mass spectrometry using an in-line sol-gel concentrator for the determination of methionine enkephalin in cerebrospinal fluid. Talanta 78, 638-642 (2009).

\section{- Metabolomics by CE-MS}

- Ramautar R, Demirci A, de Jong GJ. Capillary electrophoresis in metabolomics. Trends Anal. Chem. 25, 455-466 (2006).

- Ramautar R, Somsen GW, de Jong GJ. Direct sample injection for capillary electrophoretic determination of organic acids in cerebrospinal fluid. Anal. Bioanal. Chem. 387, 293-301 (2007).

- Ramautar R, Mayboroda OA, Deelder AM, Somsen GW, de Jong GJ. Metabolic analysis of body fluids by capillary electrophoresis using noncovalently coated capillaries. J. Chromatogr. B 871, 370-374 (2008).

- Ramautar R, Mayboroda OA, Derks R et al. CE-TOF-MS using noncovalently bilayer-coated capillaries for the analysis of amino acids in human urine. Electrophoresis 29, 2714-2722 (2008). 
- Ramautar R, Somsen GW, de Jong GJ. CE-MS in metabolomics. Electrophoresis 30, 276-291 (2009).

- Ramautar R, van de Plas A, Nevedomskaya $\mathrm{E}$ et al. Explorative analysis of urine by capillary electrophoresis-mass spectrometry in chronic patients with complex regional pain syndrome. J. Proteome Res. 8 , 5559-5567 (2009).

\section{- Monolithic LC columns}

- Rozenbrand J, van Bennekom WP, Unger KKA, de Jong GJ. Fast LC separation of a myoglobin digest: a case study using monolithic and particulate RP 18 silica capillary columns. Anal. Bioanal. Chem. 385, 1055-61 (2006).

- Van de Meent MHM, de Jong GJ. Improvement of the liquid-chromatographic analysis of protein tryptic digests by the use of long-capillary monolithic columns with UV and MS detection. Anal. Bioanal. Chem. 388, 195-200 (2007).

- van de Meent MHM, de Jong GJ. Potential of long capillary monolithic columns for the analysis of protein digests. J. Sep. Sci. 32, 487-493 (2009). 